\title{
Tratamiento quirúrgico en hernia ventral compleja en un paciente con estatus de colostomía (restitución intestinal y reparación de pared abdominal)
}

\author{
Surgical treatment of complex ventral hernia in a patient with colostomy status \\ (colostomy takedown anastomosis and abdominal wall repair)
}

Donovan Reynoso-Saldaña*, Ricardo Reynoso-González, David Estrada-Hernández, Gregorio J. Jiménez-Gamas y Cristian D. Guillén-Reveles

Servicio de Cirugía General, Hospital Regional Tlalnepantla, Instituto de Seguridad Social del Estado de México y Municipios, Tlalnepantla de Baz, Estado de México, México

\section{Resumen}

Antecedentes: Se ha descrito la reparación de hernias ventrales y alguna otra cirugía intestinal de manera simultánea. Caso clínico: Se describe el caso de una hernia ventral compleja con pérdida de dominio, en un paciente masculino en estatus de colostomía. Se realizó infiltración de toxina botulínica más neumoperitoneo progresivo preoperatorio previo al acto quirúrgico, durante el cual se realizó restitución mecánica del tránsito intestinal y hernioplastia ventral exitosamente. Conclusiones: En pacientes seleccionados, la reparación de la hernia ventral compleja, previo manejo con neumoperitoneo preoperatorio y restitución del tránsito intestinal, puede realizarse en un solo procedimiento quirúrgico, considerando las condiciones clínicas del paciente y la experiencia del cirujano.

Palabras clave: Neumoperitoneo. Hernia ventral. Hernioplastia. Colostomía.

\begin{abstract}
Background: Simultaneous ventral hernia repair with another intestinal surgery have been described. Case report: $A$ case of complex ventral hernia in a patient with colostomy status is described. Infiltration of botulinic toxin and progressive preoperative pneumoperitoneum was performed successfully prior to the surgical procedure during which mechanic restitution of gastrointestinal and ventral hernioplasty. Conclusions: In selected patients, complex ventral hernia repair, with prior progressive preoperative pneumoperitoneum, and restitution of gastrointestinal tract can be done in a single surgical procedure, considering the patient's clinical conditions and the surgeon's experience.
\end{abstract}

Key words: Pneumoperitoneum. Ventral hernia. Hernioplasty. Colostomy. 


\section{Introducción}

Dentro de los criterios para establecer una hernia compleja se incluyen una hernia mayor de $10 \mathrm{~cm}$ con pérdida de dominio en más del $20 \%$ que puede estar acompañada de infección o alguna otra condición, y una hernia en la que no es posible el cierre primario sin la previa separación de componentes ${ }^{1}$. La prevalencia reportada de hernias paraestomales varía entre el $0 \%$ y el $48 \% 2$; sin embargo, no está bien establecida la frecuencia de hernias ventrales complejas en pacientes con colostomía, ya que no es una condición reportada con frecuencia, posiblemente porque se resuelven en distintos procedimientos quirúrgicos.

Tanto la aplicación de toxina botulínica de tipo A como de neumoperitoneo progresivo preoperatorio han demostrado ser eficaces para el tratamiento de las hernias ventrales gigantes con pérdida de dominio, ya que facilitan el cierre de los grandes defectos ${ }^{3}$. Por otro lado, se han descrito casos de cierre simultáneo de defectos con anastomosis, donde se plantea que si se realizan ambos procedimientos con la técnica adecuada acorde a cada uno, con los cuidados perioperatorios propios de cualquier cirugía mayor, no debe existir inconveniente en la realización de ambos en un único acto quirúrgico ${ }^{4}$.

\section{Caso clínico}

Varón de 77 años con antecedente de diabetes mellitus tipo 2 de 4 años de evolución en tratamiento con metformina $850 \mathrm{mg} / 12 \mathrm{~h}$ por vía oral, hipertensión arterial sistémica de 4 años de evolución en tratamiento con losartán $50 \mathrm{mg} / 12 \mathrm{~h}$ por vía oral, y enfermedad pulmonar obstructiva crónica de 2 años de evolución en tratamiento con salmeterol/fluticasona 100/50 $\mu \mathrm{g} / 12 \mathrm{~h}$. Tiene antecedente quirúrgico de laparotomía exploradora y procedimiento de Hartmann secundario a enfermedad diverticular complicada hace 1 año.

Acude referido desde una unidad de medicina familiar por aumento de volumen a nivel de la línea media abdominal. En la exploración física se encuentra el abdomen con un defecto de aproximadamente $20 \times 20 \mathrm{~cm}$ con saco extenso con pérdida de dominio, así como hernia paraestomal con estoma prolapsado, sin datos de irritación peritoneal (Fig. 1).

Se realiza una tomografía contrastada (Fig. 2), en la que se observa solución de continuidad entre los

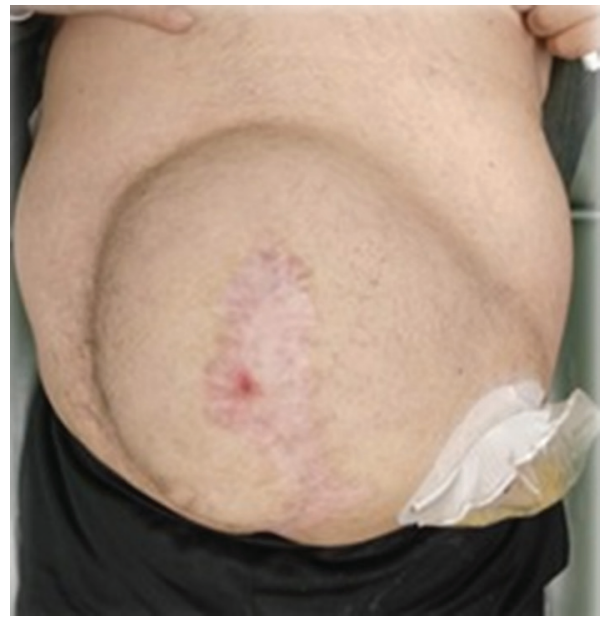

Figura 1. Hernia ventral compleja.

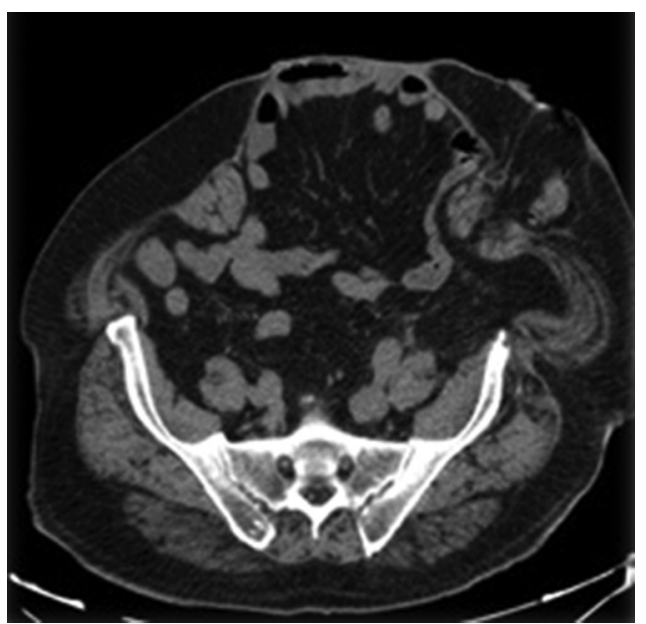

Figura 2. Imagen de tomografía abdominal que muestra una hernia ventral compleja.

músculos rectos de $24 \times 17 \times 15 \mathrm{~mm}$ con atenuación de $60 \mathrm{UH}$.

Se realiza una infiltración de $100 \mathrm{UI}$ de toxina botulínica tipo A de cada lado de la pared abdominal y se coloca un catéter trilumen en punto de Palmer para administración de neumoperitoneo progresivo preoperatorio, con control tomográfico, en el que se observan los músculos rectos retraídos con una distancia de $185 \mathrm{~mm}$ en reposo y de hasta $203 \mathrm{~mm}$ en Valsalva. En la pared lateral izquierda del abdomen se observa un defecto de pared con anillo de $31 \mathrm{~mm}$ y saco de 63 y $79 \mathrm{~mm}$ (Fig. 3).

Veintiún días tras el manejo preoperatorio con neumoperitoneo, se lleva a cabo el procedimiento quirúrgico. Se realiza una incisión en la línea media 


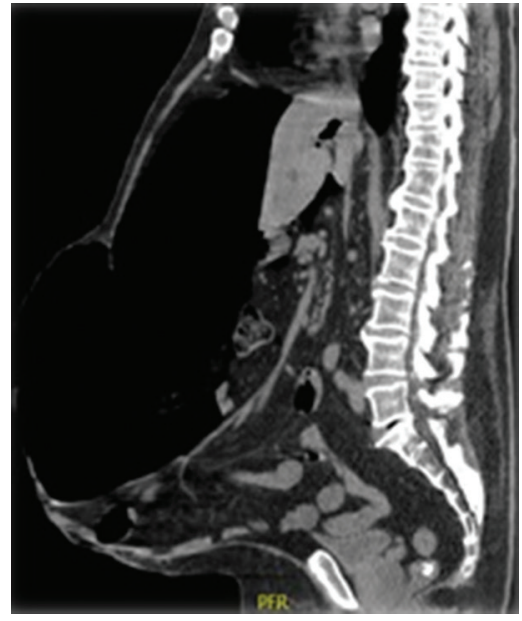

Figura 3. Imagen de tomografía abdominal que muestra el neumoperitoneo.

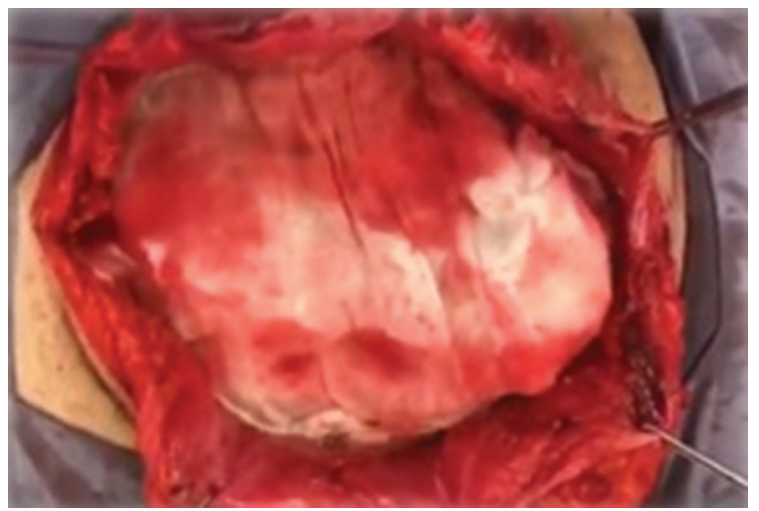

Figura 4. Disección, apertura y localización de los bordes aponeuróticos.

supraumbilical e infraumbilical, se identifica el saco herniario y se continúa con su disección y apertura, se realiza adherenciólisis asa-asa intestinal y saco-asas intestinales (Fig. 4), se localizan los bordes aponeuróticos y la aponeurosis del oblicuo mayor y se realiza la sección de esta, obteniendo un gran avance aponeurótico para la reconstrucción de la pared abdominal (Fig. 5). Posteriormente se desmanteló la colostomía para realizar una restitución intestinal mecánica. Se continuó la resección del saco y el cierre de la aponeurosis, con colocación supra-aponeurótica de malla de polipropileno de $30 \times 30 \mathrm{~cm}$ fijándola con puntos simples hacia la aponeurosis. Se resecó el excedente de piel con incisión de flor de lis, se colocaron dos drenajes cerrados (Biovac ${ }^{\mathrm{TM}}$ ) y se afrontó la piel con puntos Ashiff.

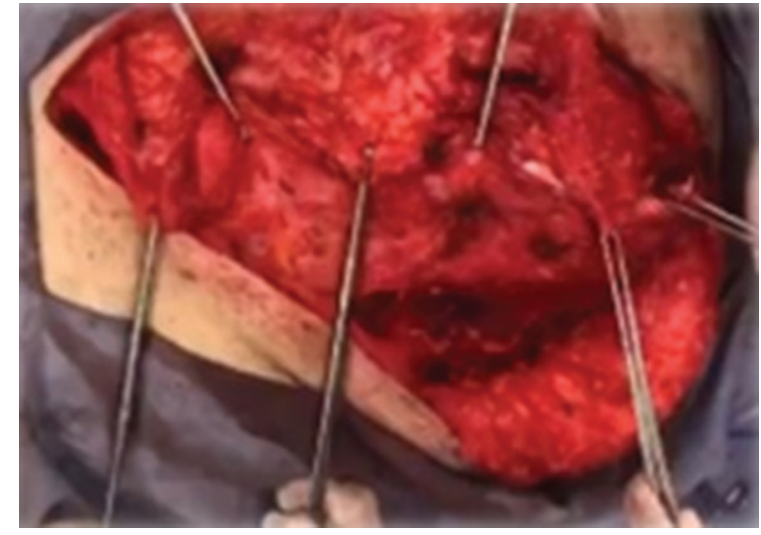

Figura 5. Técnica de separación anatómica de componentes de Carbonell nivel I. Sección de la aponeurosis del oblicuo mayor, obteniendo un gran avance para el cierre aponeurótico.

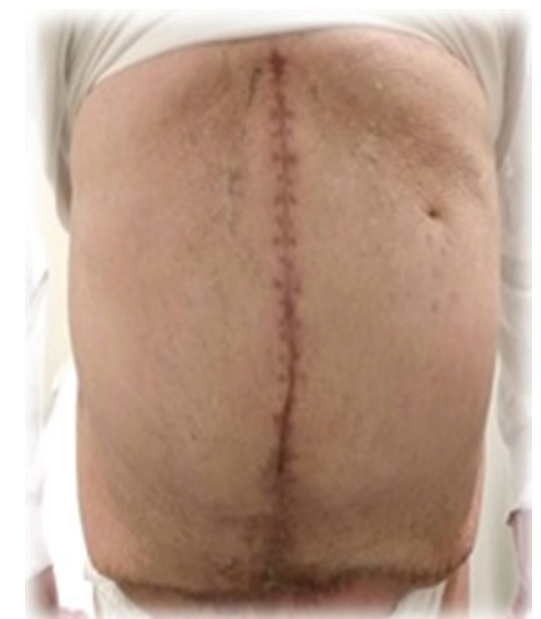

Figura 6. Seguimiento del paciente a 3 meses del evento quirúrgico.

Se dio seguimiento al paciente cada 7 días durante los primeros 2 meses. A los 21 días se retiraron los drenajes considerando un gasto $<50 \mathrm{ml}$ en 24 horas. Posteriormente se valoró a los 3, 6 y 12 meses, sin complicaciones ni recidiva (Fig. 6).

\section{Discusión}

Previamente se ha descrito la reparación de hernias complejas con una restitución simultánea del tránsito intestinal con y sin uso de neumoperitoneo con el fin de lograr la elongación de los músculos de la pared abdominal y la lisis de adherencias ${ }^{5}$. En nuestro caso usamos además toxina botulínica 21 días antes del procedimiento, logrando una adecuada relajación de la pared, lo que permitió durante la cirugía realizar 
una plastia de pared abdominal sin tensión, lo cual consideramos un factor importante para la evolución del paciente en relación a la plastia y en segunda instancia a la anastomosis intestinal ${ }^{6}$. Un estudio retrospectivo en el que se analizaron 18 pacientes sometidos a reparación de hernia incisional más restitución del tránsito intestinal observó fuga de la anastomosis en cuatro pacientes $(18 \%)^{7}$, lo que concuerda con series en las que se realizaba la reparación de una hernia compleja con reconstrucción y manejo de fístula enterocutánea ${ }^{8}$. Cabe aclarar que no se puede comparar directamente con el caso presentado, pues en ninguno de los dos estudios se menciona el uso de neumoperitoneo preoperatorio ni de toxina botulínica, que puede ser un factor en el éxito quirúrgico y, sin embargo, no ha sido estudiado en este tipo de situaciones. En el caso presentado se consideraron factores para determinar la confección de la anastomosis intestinal que el paciente presentara unos adecuados valores de hemoglobina ( $>11 \mathrm{mg} / \mathrm{dl}$ ), un buen estado nutricional, un IMC $<30$ y no tuviera antecedente de tabaquismo. Debido a que actualmente no existen recomendaciones precisas en cuanto al manejo de las hernias complejas con pérdida de dominio en pacientes con estatus de colostomía, la decisión de las técnicas preoperatorias previamente mencionadas se basó en la experiencia del equipo quirúrgico en la resolución de hernias complejas.

En nuestro caso se obtuvieron adecuados resultados en el paciente, por lo que pudo egresar sin complicaciones, y si bien se requieren estudios más grandes para emitir algún tipo de recomendación en este tipo de casos, se conoce que el $32.2 \%$ en que se realiza alguna otra cirugía adicional presentan recurrencia al realizar alguna cirugía adicional abdominal $^{9}$, por lo que es necesario tener un adecuado protocolo de selección de pacientes, que deben tener un adecuado control de cualquier comorbilidad, como en el caso expuesto, y la corrección de cualquier factor modificable que pudiera aumentar el riesgo quirúrgico per se.

\section{Conclusiones}

Es posible el manejo simultáneo de la hernia ventral compleja (previo neumoperitoneo progresivo más aplicación de toxina botulínica) y la restitución del tránsito intestinal en pacientes seleccionados, con un adecuado protocolo prequirúrgico y realizado por un equipo con experiencia en el manejo de ambas condiciones aisladas, considerando los factores de riesgo del paciente, que en el caso expuesto fueron la presencia de un buen estado nutricional, hemoglobina $>11 \mathrm{mg} / \mathrm{dl}$, un IMC $<30$ y sin antecedente de tabaquismo, lo que permitirá que no se presenten complicaciones en relación a la anastomosis intestinal. Es importante señalar que no existen estudios que evalúen este tipo de escenarios, por lo que con base en la evidencia actual y la experiencia del equipo quirúrgico se debe considerar realizar una reconstrucción de la pared abdominal sin tensión, con empleo de toxina botulínica y neumoperitoneo preoperatorio. De esta forma se puede disminuir el riesgo de las principales complicaciones de estos procedimientos en combinación, como son la fuga de la anastomosis intestinal y la infección del material protésico.

\section{Agradecimientos}

Los autores agradecen al servicio de cirugía general del Hospital Regional Tlalnepantla del Instituto de Seguridad Social del Estado de México y Municipios por el apoyo brindado.

\section{Responsabilidades éticas}

Protección de personas y animales. Los autores declaran que para esta investigación no se han realizado experimentos en seres humanos ni en animales.

Confidencialidad de los datos. Los autores declaran que han seguido los protocolos de su centro de trabajo sobre la publicación de datos de pacientes.

Derecho a la privacidad y consentimiento informado. Los autores han obtenido el consentimiento informado de los pacientes y/o sujetos referidos en el artículo. Este documento obra en poder del autor de correspondencia.

\section{Financiamiento}

Los autores declaran no haber recibido financiamiento para llevar a cabo este artículo.

\section{Conflicto de intereses}

Los autores declaran no tener ningún conflicto de intereses. 


\section{Bibliografía}

1. Slater NJ, Montgomery A, Berrevoet F, Carbonell AM, Chang A Franklin $\mathrm{M}$, et al. Criteria for definition of a complex abdominal wall hernia. Hernia. 2014;18:7-17.

2. Shah NR, Craft RO, Harold KL. Parastomal hernia repair. Surg Clin North Am. 2013;93:1185-98.

3. Benavides D, Solorzano J, García M, Manrique M, Pina M, Gómez Z, et al. Asociación de neumoperitoneo progresivo preoperatorio y separación posterior de componentes con liberación del músculo transverso en el tratamiento de una eventración gigante con pérdida de derecho a domicilio. Rev Hisp Hernia. 2015;5:115-9.

4. Hernández López A. Tratamiento simultáneo de plastía de pared con malla y cierre intestinal. Cir Gen. 2004;26:242-7.
5. Cuenca Torres O, Ortiz J, Ferreira R, Sosa C, González E, Pederzoli R. Reparación de defectos complejos de la pared abdominal y cirugía intestinal en un tiempo. Cir Parag. 2016;40:13-7.

6. Trallero Anoro M, Carbonell Tatay F, García Fadrique A, Caballero Soto M, Carreño Suárez O, Campos Máñez J, et al. Reparación en dos tiempos de triple hernia (perineal, paraestomal y línea media) con uso de toxina botulínica en pared abdominal. Rev Hisp Hernia. 2018;6:91-5.

7. Baastrup NN, Hartwig MFS, Krarup PM, Jorgensen LN, Jensen KK. Anastomotic leakage after stoma reversal combined with incisional hernia repair. World J Surg. 2019;43:988-97.

8. Krpata DM, Stein SL, Eston M, Ermlich B, Blatnik JA, Novitsky YW, et al. Outcomes of simultaneous large complex abdominal wall reconstruction and enterocutaneous fistula takedown. Am J Surg. 2013;205:354-9.

9. De Vries FEE, Hodgkinson JD, Claessen JJM, van Ruler O, Leo CA, Maeda $Y$, et al. Long-term outcomes after contaminated complex abdominal wall reconstruction. Hernia. 2020;24:459-68. 